\title{
Cometary plasma response to interplanetary corotating interaction regions during 2016 June-September: a quantitative study by the Rosetta Plasma Consortium
}

\author{
Rajkumar Hajra, ${ }^{1 \star}$ Pierre Henri, ${ }^{1}$ Minna Myllys, ${ }^{1}$ Kevin L. Héritier, ${ }^{2}$ Marina Galand, ${ }^{2}$ \\ Cyril Simon Wedlund, ${ }^{3}$ Hugo Breuillard, ${ }^{1,4}$ Etienne Behar, ${ }^{5}$ Niklas J. T. Edberg, ${ }^{6}$ \\ Charlotte Goetz, ${ }^{7}$ Hans Nilsson, ${ }^{5}$ Anders I. Eriksson, ${ }^{6}$ Raymond Goldstein, ${ }^{8}$ \\ Bruce T. Tsurutani, ${ }^{9}$ Jerome Moré, ${ }^{1}$ Xavier Vallières ${ }^{1}$ and Gaetan Wattieaux ${ }^{10}$ \\ ${ }^{1}$ Laboratoire de Physique et Chimie de l'Environnement et de l'Espace (LPC2E), CNRS, 45071 F-Orléans, France \\ ${ }^{2}$ Department of Physics, Imperial College London, Prince Consort Road, London, SW7 2AZ, UK \\ ${ }^{3}$ Department of Physics, University of Oslo, Box 1048 Blindern, 0316 Oslo, Norway \\ ${ }^{4}$ Laboratoire de Physique des Plasmas, Ecole Polytechnique/CNRS/Sorbonne Université, 4 Place Jussieu, F-75252 Paris, France \\ ${ }^{5}$ Swedish Institute of Space Physics, PO Box 812, SE-981 28 Kiruna, Sweden \\ ${ }^{6}$ Institutet för rymdfysik, Ångstrom Laboratory, Lagerhyddsvagen 1, SE-75237 Uppsala, Sweden \\ ${ }^{7}$ Institut für Geophysik und extraterrestrische Physik, TU Braunschweig, Mendelssohnstr. 3, D-38106 Braunschweig, Germany \\ ${ }^{8}$ Southwest Research Institute, PO Drawer 28510, San Antonio, TX 78228-0510, USA \\ ${ }^{9}$ Jet Propulsion Laboratory, California Institute of Technology, 4800 Oak Grove Drive, Pasadena, CA 91109, USA \\ ${ }^{10}$ LAPLACE, Universite de Toulouse, CNRS, F-31062 Toulouse, France
}

Accepted 2018 August 7. Received 2018 July 25; in original form 2018 May 16

\begin{abstract}
Four interplanetary corotating interaction regions (CIRs) were identified during 2016 JuneSeptember by the Rosetta Plasma Consortium (RPC) monitoring in situ the plasma environment of the comet 67P/Churyumov-Gerasimenko (67P) at heliocentric distances of $\sim 3-3.8$ au. The CIRs, formed in the interface region between low- and high-speed solar wind streams with speeds of $\sim 320-400 \mathrm{~km} \mathrm{~s}^{-1}$ and $\sim 580-640 \mathrm{~km} \mathrm{~s}^{-1}$, respectively, are characterized by relative increases in solar wind proton density by factors of $\sim 13-29$, in proton temperature by $\sim 7-29$, and in magnetic field by $\sim 1-4$ with respect to the pre-CIR values. The CIR boundaries are well defined with interplanetary discontinuities. Out of 10 discontinuities, four are determined to be forward waves and five are reverse waves, propagating at $\sim 5-92$ percent of the magnetosonic speed at angles of $\sim 20^{\circ}-87^{\circ}$ relative to ambient magnetic field. Only one is identified to be a quasi-parallel forward shock with magnetosonic Mach number of $\sim 1.48$ and shock normal angle of $\sim 41^{\circ}$. The cometary ionosphere response was monitored by Rosetta from cometocentric distances of $\sim 4-30 \mathrm{~km}$. A quiet time plasma density map was developed by considering dependences on cometary latitude, longitude, and cometocentric distance of Rosetta observations before and after each of the CIR intervals. The CIRs lead to plasma density enhancements of $\sim 500-1000$ per cent with respect to the quiet time reference level. Ionospheric modelling shows that increased ionization rate due to enhanced ionizing ( $>12-200 \mathrm{eV}$ ) electron impact is the prime cause of the large cometary plasma density enhancements during the CIRs. Plausible origin mechanisms of the cometary ionizing electron enhancements are discussed.
\end{abstract}

Key words: methods: data analysis - methods: observational - Sun: rotation - solar windcomets: general - comets: individual: 67P/Churyumov-Gerasimenko.

\section{INTRODUCTION}

^E-mail: rajkumarhajra@yahoo.co.in

The cometary plasma and magnetic field dynamics are primarily due to solar heating and the interaction with the solar wind. While 
the solar heating is slowly varying in time, the solar wind itself can be very dynamic. For example coronal mass ejections (CMEs) associated with solar flares, and high-speed streams (HSSs) and corotating interaction regions (CIRs) associated with coronal holes have high plasma density features that can cause sudden compressions of the cometary plasma environment. The aim of this work is to study the characteristics and impacts of interplanetary CIRs on the near-cometary plasma environment at large heliocentric distances. It is based on the plasma and magnetic field measurements by instruments onboard the Rosetta spacecraft (Glassmeier et al. 2007a) around comet 67P/Churyumov-Gerasimenko (hereafter referred to as 67P, Churyumov \& Gerasimenko 1972) during 2016 June through September.

The CIRs are formed in interplanetary space owing to the interaction between HSSs and the background quiet/low-speed streams (Siscoe 1972; Collard \& Wolfe 1974; Smith \& Wolfe 1976; Pizzo 1985; Balogh et al. 1999). As coronal holes are long-lived structures, the interaction regions may 'corotate' and reappear with solar rotation ( $\sim 25 \mathrm{~d})$. At large heliocentric distances ( $\geq 3 \mathrm{au})$ the leading and trailing edges of the CIRs are marked by interplanetary forward and reverse shocks, respectively (Parker 1963; Sonett \& Colburn 1965; Smith \& Wolfe 1976; Burlaga, Mish \& Whang 1990; Tsurutani et al. 1995; Echer, Tsurutani \& Guarnieri 2010). However, as the shock formation occurs due to the non-linear steepening of waves that requires several non-linear steepening times, most CIRs do not have shocks at 1 au (Neugebauer \& Snyder 1966; Chao 1973; Intriligator \& Neugebauer 1975; Smith \& Wolfe 1976). The CIRs are characterized by compressed plasma and magnetic field regions. These are known to be geoeffective causing weak to moderate geomagnetic storms, and intense, long-duration auroral activities at the Earth (Tsurutani \& Gonzalez 1987; Tsurutani et al. 2006; Hajra et al. 2013, 2014a,c,b, 2015a,b, 2017a; Souza et al. 2016, 2018; Mendes et al. 2017; Guarnieri et al. 2018; Hajra \& Tsurutani 2018a). They result from magnetic reconnection (Dungey 1961) between the southward component of CIR magnetic fields and the Earth's dayside magnetopause fields. While CIR impacts on the Earth's magnetosphere-ionosphere system are well studied, less explored are the impacts on non-magnetized solar system bodies, like Mars, Venus, and comets. The ionospheric escape rates of Mars and Venus are reported to increase during CIR events (Dubinin et al. 2008; Edberg et al. 2011 and references therein). Edberg et al. (2016) reported the first in situ study of comet 67P ionospheric response to CIR events. The CIRs were shown to cause enhancements in the cometary plasma density, ionizing electron fluxes, and magnetic field strengths at $\sim 3$ au from the Sun during the pre-perihelion phase of the comet. While the exact mechanism of the cometary plasma enhancements could not be identified in that study, four potential mechanisms were suggested, namely, the plasma environment compression, increase in electron-impact ionization, increase in charge exchange processes, and acceleration of mass-loaded plasma towards the comet nucleus. One of the aims of this study is to discriminate between the dominant process(es).

In this work, the response of the cometary ionosphere during CIRs are explored to extract and quantify the CIR contribution on the cometary plasma variability. In this study, the comet is in the post-perihelion phase of its solar orbit. Comparison has therefore been undertaken with the study of Edberg et al. (2016) when the comet was in the pre-perihelion phase and at almost similar heliocentric distances. It may be mentioned that around the perihelion, in situ solar wind measurements by Rosetta, and thus a direct detection of CIRs, was not possible due to formation of a near-nucleus solar wind cavity void of any solar wind ions
(Behar et al. 2017; Nilsson et al. 2017). In addition to the impact of the CIRs on the cometary plasma, the features of the interplanetary characteristics of the CIRs in terms of the solar wind proton density, speed, temperature, and magnetic field intensity are studied in details. This allows a deeper insight and a better understanding of the interplanetary CIR evolution at large heliocentric distances.

The paper is organized as follows: In Section 2, we give an overview of the Rosetta spacecraft observations during 2016 JuneSeptember followed by a case-by-case study on the CIR events. The results are discussed in Section 3 and the main findings are summarized in Section 4. Few final comments are added in Section 5.

\section{DATA ANALYSES AND RESULTS}

For this study, we focus on the time interval from 2016 June 1 to 2016 September 30, during the descending-to-minimum phase of the solar cycle 24 , characterized by large and well-defined coronal holes in the polar and equatorial regions of the Sun. Comet $67 \mathrm{P}$, orbiting the Sun at heliocentric distance $r_{\mathrm{h}}$ between $\sim 3$ and 3.8 au during this interval, encountered with four consecutive HSSs emanating from coronal holes. The interaction between the solar wind and the inner cometary plasma environment/induced magnetosphere is monitored by five instruments of the Rosetta Plasma Consortium (RPC, Carr et al. 2007) onboard the Rosetta spacecraft from the cometocentric distance $r_{\mathrm{c}}$ between $\sim 4$ and $30 \mathrm{~km}$. In the following subsections near-cometary plasma and magnetic field measurements during the CIRs are described and discussed.

\subsection{RPC observations of CIRs during 2016 June-September: an overview}

Fig. 1 shows measurements by different RPC instruments during the last four months of the Rosetta mission operations around comet 67P, from 2016 June 1 through 2016 September 30 (153-274 d of year/DOY 2016). While 67P moved gradually from the heliocentric distance $r_{\mathrm{h}}$ of $\sim 3.1$ au to $\sim 3.8 \mathrm{au}$, Rosetta remained in the inner cometary ionosphere/induced magnetosphere, mostly within $30 \mathrm{~km}$ from the comet nucleus (Fig. 1i). The ion energy spectrogram obtained from the Ion and Electron Sensor of the RPC (RPC-IES, Burch et al. 2007) shows clear signature of solar wind protons $\left(\mathrm{H}^{+}\right)$ and alpha particles $\left(\mathrm{He}^{++}\right)$, energy varying between $\sim 700 \mathrm{eV}$ and $\sim 4 \mathrm{keV}$ (Fig. 1e). The solar wind proton speed (Vp), density (Np), and temperature (Tp) are estimated by fitting a Maxwellian distribution to RPC-IES proton data. The estimated parameters are shown in Figs $1 \mathrm{a}, \mathrm{b}$, and c, respectively (blue points). The $\mathrm{Vp}$ and $\mathrm{Np}$ are also estimated by integrating the plasma moments of order 0 and 1 obtained from the Ion Composition Analyzer (RPC-ICA, Nilsson et al. 2007) that measures the ion distribution in the energy range of $10 \mathrm{eV}-40 \mathrm{keV}$ (Figs 1a and b, red points). Although the estimations have different time resolutions, both the estimates for $\mathrm{Vp}$ show a similar temporal behaviour. However, the estimated parameters from RPC-IES data show large scatters (uncertainties) during certain time intervals. The scatters are due to the fitting procedure and are related to the time bins when the RPC-IES proton distribution fits poorly to Maxwellian.

From the variation of $\mathrm{Vp}$, four HSSs can be identified with peak $\mathrm{Vp}$ of $\sim 590 \mathrm{~km} \mathrm{~s}^{-1}, 580 \mathrm{~km} \mathrm{~s}^{-1}, 640 \mathrm{~km} \mathrm{~s}^{-1}$, and $590 \mathrm{~km} \mathrm{~s}^{-1}$ at $\sim 1030$ UT on day 168 , at $\sim 1030$ UT on day 193, at $\sim 2130$ UT on day 219 , and at $\sim 1130$ UT on day 247 , respectively (Fig. 1a). These are significantly less than $\sim 750-800 \mathrm{~km} \mathrm{~s}^{-1}$ speeds observed by Ulysses at $\sim 3.7$ au distance over the solar poles (Phillips et al. 1994). This could either mean that Rosetta is seeing the edges of the 


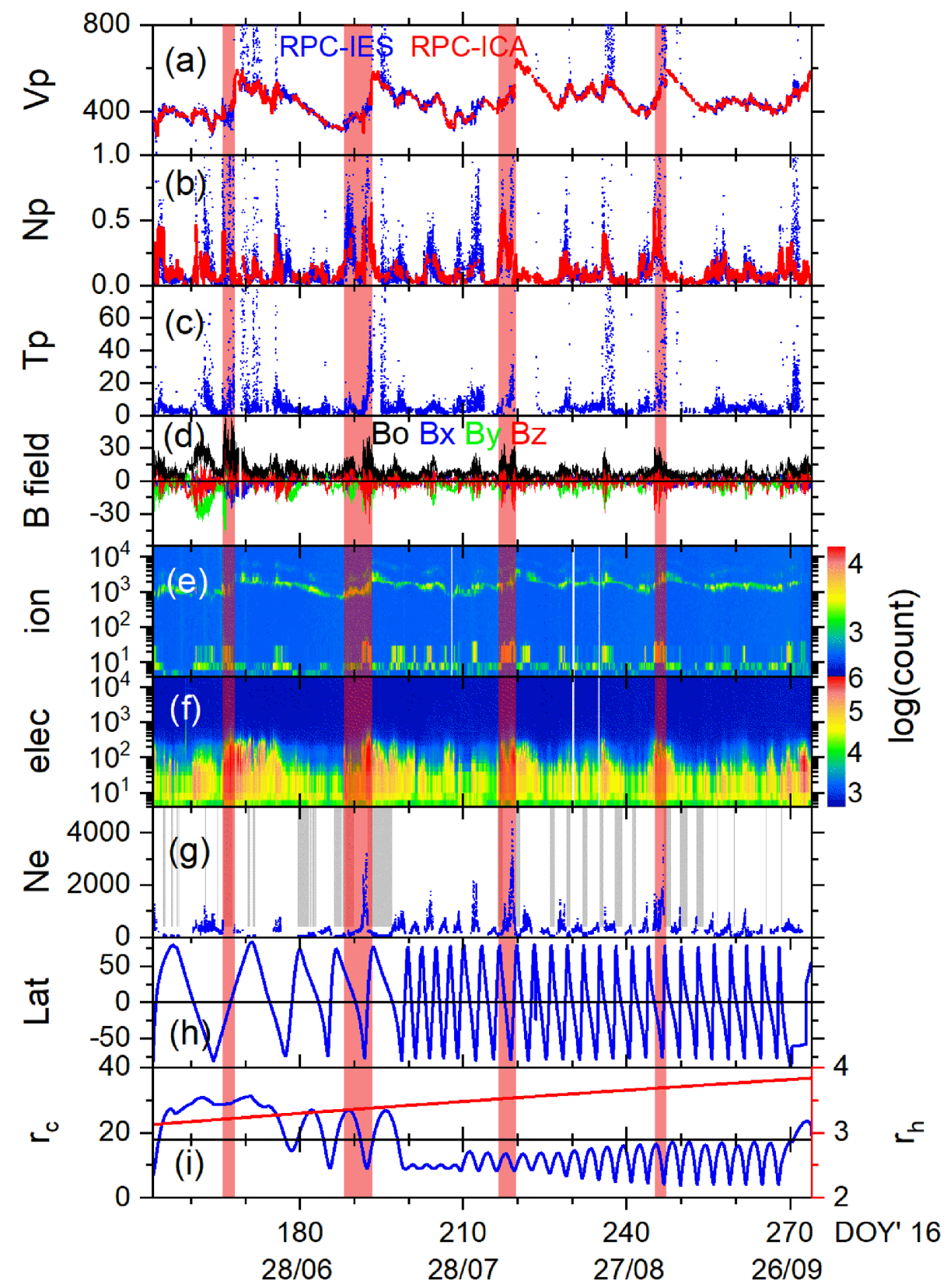

Figure 1. RPC observations during 2016 June through September. From top to bottom, the panels show (a) solar wind proton speed $\mathrm{Vp}$ (in $\mathrm{km} \mathrm{s}^{-1}$ ) measured by RPC-IES (blue) and RPC-ICA (red), (b) solar wind proton density Np (in $\mathrm{cm}^{-3}$ ) measured by RPC-IES (blue) and RPC-ICA (red), (c) solar wind proton temperature Tp (in eV) measured by RPC-IES, (d) magnetic field amplitude Bo (in nT, black), and Bx (blue), By (green), Bz (red) components in CSEQ coordinate system from RPC-MAG, (e) RPC-IES ion spectrogram (legend on the right), (f) RPC-IES electron spectrogram (legend on the right), (g) cometary plasma density $\mathrm{Ne}\left(\mathrm{in}_{\mathrm{cm}}^{-3}\right.$ ) measured by RPC-MIP, the grey shadings indicating inaccessible densities associated with measurement limits in LDL operational mode, (h) latitude (in degree), (i) cometocentric distance $r_{\mathrm{c}}$ (in km) of Rosetta (blue) and heliocentric distance $r_{\mathrm{h}}$ (in au) of comet 67P (red), respectively. The light red shaded regions show the CIR impact intervals.

HSSs or that these have been decelerated. The consecutive HSSs are separated by $\sim 25, \sim 26.5$, and $\sim 27.4 \mathrm{~d}$, respectively. These correspond to the solar differential rotation periods at the solar equator to mid-latitude regions (Bartels 1932, 1934; Newton \& Nunn 1951; Tsurutani et al. 1982). The low-speed streams in between the HSSs had $\mathrm{Vp}$ in the range $\sim 320-400 \mathrm{~km} \mathrm{~s}^{-1}$. The HSSs likely emanated from the coronal holes observed on days 159 (June 7), 182 (June 30), 209 (July 27), and 235 (August 22), respectively, as shown in Fig. 2. The solar coronal images are taken by the Atmospheric Imaging As- sembly (AIA) telescope of NASA's Solar Dynamics Observatory (SDO) at the wavelength of $193 \AA$ (https://sdo.gsfc.nasa.gov/).

The HSSs are preceded by CIRs, whose impact intervals are marked by light red regions in Fig. 1. The solar conditions as well as the interplanetary parameters confirm that the intervals are free from any other solar events. During the CIRs, in general, both the proton density $\mathrm{Np}$ and the temperature $\mathrm{Tp}$ show large enhancements. The magnetic field components $(\mathrm{Bx}, \mathrm{By}, \mathrm{Bz})$ and amplitude $\mathrm{Bo}$ measured by the three-axial fluxgate magnetometers (RPC-MAG, 

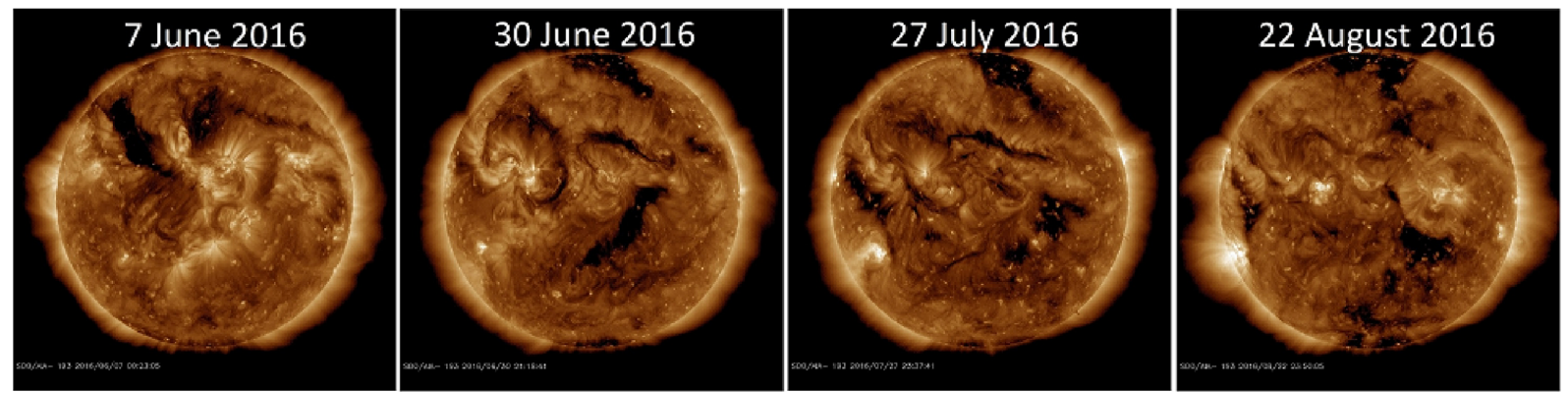

Figure 2. Solar images taken by the SDO/AIA telescope at the wavelength of $193 \AA$.

Glassmeier et al. 2007b) show magnetic field compression and polarity fluctuations (Fig. 1d). The magnetic field is expressed in the Cometocentric Solar Equatorial (CSEQ) coordinate system where the $x$-axis points towards the Sun, the $z$-axis is the component of the solar north pole that is orthogonal to the $x$-axis, and the $y$-axis completes the right-handed coordinate system. The reader should be aware of offset uncertainty of the magnetic field measurements during this interval. To constrain the error that arises from this, we add the maximum offset of $5 \mathrm{nT}$ to intervals where the field magnetitude is below $5 \mathrm{nT}$ and consider this to be the upper limit for the error.

Fig. If shows the electron energy spectrum obtained from RPCIES. It is clear that CIR impacts are followed by significant increases in the ionizing $(>12-200 \mathrm{eV})$ electron fluxes as well as increases in the cometary ion fluxes (Fig. 1e). Cometary plasma is monitored by a Mutual Impedance Probe (RPC-MIP, Trotignon et al. 2007) and two LAngmuir Probes (RPC-LAP, Eriksson et al. 2007). Fig. 1g shows the electron density Ne derived from the RPC-MIP measurements. Ne represents the total electron density including the sum of different populations, namely, the cold, warm, and ionizing populations. There are apparent increases in Ne during the CIR intervals compared to pre- and post-CIR periods.

Case-by-case analyses of the CIR interplanetary characteristics and their impacts on the cometary ionosphere/magnetosphere are presented in Sections 2.2-2.5.

\subsection{CIR event during 2016 June 13-15}

Fig. 3 shows the RPC observations during 2016 June 12 to 2016 June 21 (days 164 to 173). The CIR impact interval is well defined with discontinuities in the solar wind plasma and magnetic field variations at $\sim 1913$ UT on day 165 , and at $\sim 2326$ UT on day 167. These are marked by FW and RW on the top of the figure, representing forward and reverse waves, respectively. The CIR had a total duration of $\sim 2.18 \mathrm{~d}$ between a low-speed stream with $\mathrm{Vp}$ $\sim 375 \mathrm{~km} \mathrm{~s}^{-1}$ and an HSS with peak Vp $\sim 590 \mathrm{~km} \mathrm{~s}^{-1}$ (Fig. 3a). The FW is characterized by increases in Vp, Np, Tp, and Bo (Smith \& Wolfe 1976; Richter et al. 1985; Tsurutani et al. 2011). For the RW, Vp increased, but the other parameters (Np, Tp, and Bo) decreased with time.

To identify the nature of the discontinuities, we estimated the magnetosonic Mach numbers (MMNs) and angles of propagations of the discontinuities using the Abraham-Shrauner (1972) mixedmode method and the Rankine-Hugoniot conservation equations (Smith 1985; Tsurutani \& Lin 1985; Tsurutani et al. 2011; Hajra et al. 2016b; Hajra \& Tsurutani 2018b). We considered average values of the solar wind plasma and magnetic field parameters during about $2 \mathrm{~h}$ intervals upstream and downstream sides of the discon- tinuities. The FW and RW are found to have MMNs of $\sim 0.19$ and $\sim 0.11$, and normal angles of $\sim 26^{\circ}$ and $\sim 86^{\circ}$ relative to the upstream magnetic field, respectively. The low MMNs $(<1)$ confirm that the discontinuities were not shocks, but waves. This result implies that the FW was propagating at only $\sim 19$ per cent of the magnetosonic speed at an angle of $\sim 26^{\circ}$ relative to ambient magnetic field. On the other hand, the RW was quasi-perpendicular, propagating at $\sim 11$ per cent of the magnetosonic speed at an angle of $\sim 86^{\circ}$ relative to ambient magnetic field. We identified a total of 10 such solar wind plasma and magnetic field discontinuities around the CIRs under this study. The discontinuity characteristic parameters are listed in Table 1. We remind that the offset uncertainty of the magnetic field measurements and the uncertainties in the solar wind proton parameters may introduce errors in the above estimations.

During the CIR interval, 67P was at a heliocentric distance of $\sim 3.2$ au, while Rosetta was at a cometocentric distance of $\sim 28.6$ $29.6 \mathrm{~km}$ (Fig. 3i), moving from the southern hemisphere towards the north (Fig. 3h). The CIR/FW impact coincides with enhancement in the magnetic field amplitude Bo from $\sim 13-15 \mathrm{nT}$ to $\sim 62 \mathrm{nT}$, and polarity reversal in By and Bz components (Fig. 3d). This magnetic field polarity reversal within the low-speed wind is characteristic of a heliospheric current sheet (HCS, Ness \& Wilcox 1964; Smith, Tsurutani \& Rosenberg 1978). This is accompanied by neighboring high-density cold plasma, known as heliospheric plasma sheet (HPS, Winterhalter et al. 1994).

The CIR impact is characterized by a large spread (compared to pre-CIR period) in solar wind proton energy spectrum (Fig. 3e), resulting in large ranges of variations in $\mathrm{Vp}, \mathrm{Np}$, and $\mathrm{Tp}$. The compressed proton density changes from $\sim 0.03 \mathrm{~cm}^{-3}$ to $\sim 0.43 \mathrm{~cm}^{-3}$, a factor of $\sim 13$ increase relative to the pre-CIR value. Although $\mathrm{Tp}$ exhibits large uncertainty in measurement, it was $\sim 2.6 \mathrm{eV}$ before CIR impact, and increased abruptly to $\sim 19.5 \mathrm{eV}$ after the impact.

Cometary ion $(\sim 4-20 \mathrm{eV})$ flux increases may be noted from the RPC-IES measurements (Fig. 3e) owing to increase in the negative spacecraft potential (not shown). Another prominent feature of the CIR impact is large increase in ionizing $(>12-200 \mathrm{eV})$ electron fluxes, which continues $\sim 1.5 \mathrm{~d}$ after the CIR terminates (Fig. 3f). About 3 orders of magnitude increase was recorded during the CIR interval relative to the pre-CIR value.

During the CIR interval, RPC-MIP was mostly operated in long Debye length (LDL) mode, while it was operated in short Debye length (SDL) mode during pre- and post-CIR intervals (see Trotignon et al. 2007 for a detailed description of SDL and LDL modes). SDL mode is used to measure high densities of cold plasma while LDL can measure only low electron densities, usually $<300 \mathrm{~cm}^{-3}$ (Fig. 3g). Estimated Ne before and after the CIR varied between $\sim 200$ and $900 \mathrm{~cm}^{-3}$. As RPC-MIP can only estimate plasma density below $\sim 300 \mathrm{~cm}^{-3}$ in the LDL mode (shown by grey 


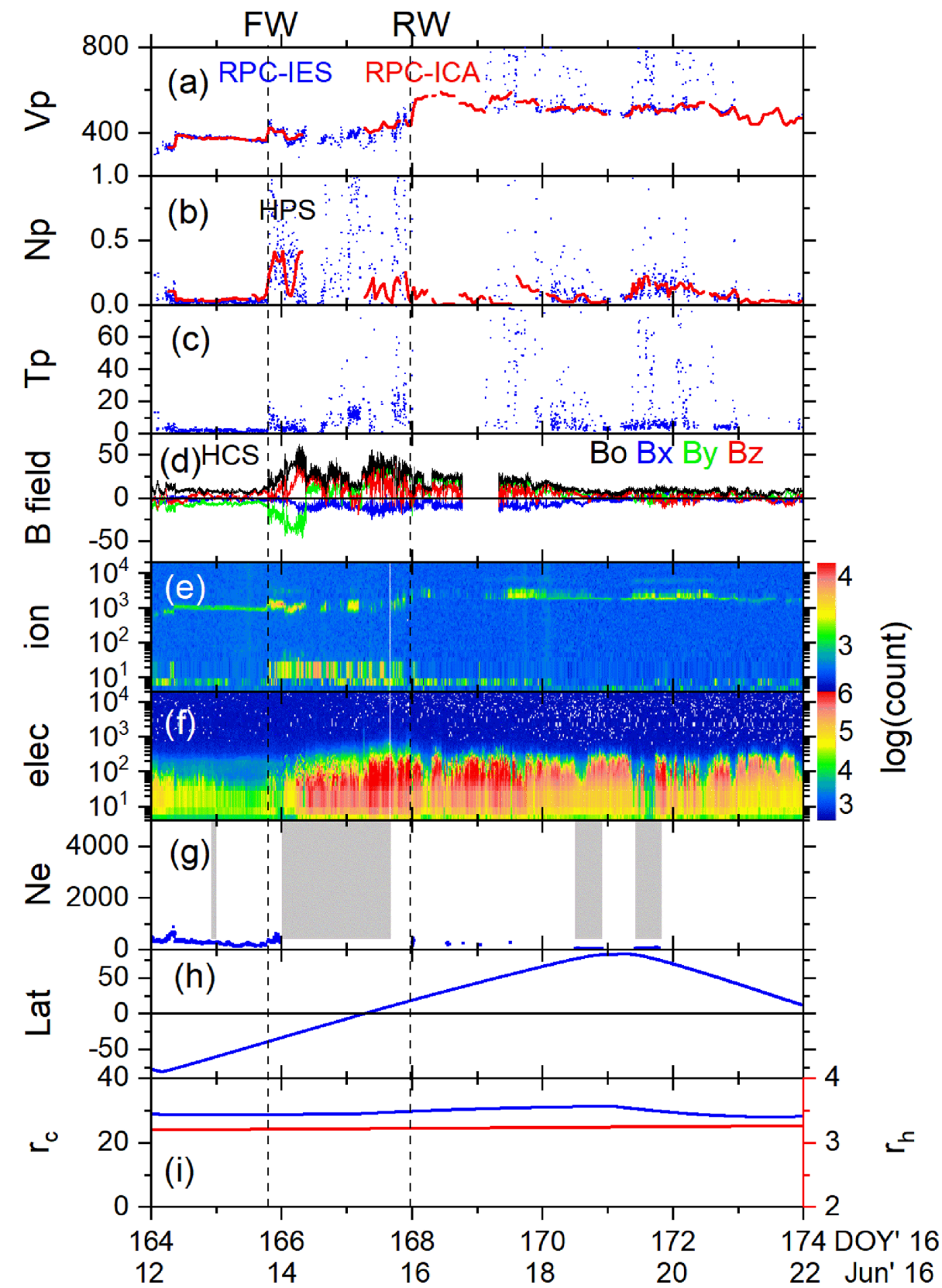

Figure 3. RPC observations for CIR during 2016 June 13-15. The panels are in the same format as in Fig. 1. FW and RW are shown by vertical dashed lines.

Table 1. Characteristics of the interplanetary discontinuities at the CIR boundaries as observed by RPC.

\begin{tabular}{lccc}
\hline Time & Type & Angle & MMN \\
\hline 1913 UT June 13 (Fig. 3) & FW & $26^{\circ}$ & 0.19 \\
2326 UT June 15 (Fig. 3) & RW & $86^{\circ}$ & 0.11 \\
0141 UT July 06 (Fig. 4) & FW & $29^{\circ}$ & 0.36 \\
0420 UT July 08 (Fig. 4) & RW & $87^{\circ}$ & 0.05 \\
1702 UT July 09 (Fig. 4) & FS & $41^{\circ}$ & 1.48 \\
0407 UT July 11 (Fig. 4) & RW & $80^{\circ}$ & 0.92 \\
0925 UT Aug 03 (Fig. 5) & FW & $68^{\circ}$ & 0.13 \\
1234 UT Aug 06 (Fig. 5) & RW & $86^{\circ}$ & 0.13 \\
0129 UT Sep 01 (Fig. 6) & FW & $20^{\circ}$ & 0.59 \\
0623 UT Sep 03 (Fig. 6) & RW & $63^{\circ}$ & 0.76 \\
\hline
\end{tabular}

shading in Fig. $3 \mathrm{~g}$ ), an $\mathrm{Ne}$ data gap during the CIR interval suggests that Ne was likely to be $>300 \mathrm{~cm}^{-3}$ during the CIR. However, the exact plasma density response to this CIR cannot be inferred.

\subsection{CIR event during 2016 July 6-11}

Fig. 4 shows an interesting 'double CIR' structure from 2016 July 6 to 2016 June 11 (days 188 to 193) between a low-speed $\left(\sim 330 \mathrm{~km} \mathrm{~s}^{-1}\right)$ stream and an HSS $\left(\sim 580 \mathrm{~km} \mathrm{~s}^{-1}\right)$. This interval is characterized by an FW at $\sim 0141$ UT on day 188 , an FS at $\sim 1702$ UT on day 191, and two RWs at $\sim 0420$ UT on day 190 and at $\sim 0407$ UT on day 193 (Table 1).

During the entire CIR interval of $\sim 5.10 \mathrm{~d}, 67 \mathrm{P}$ moved slowly from the heliocentric distance of $\sim 3.3$ au to $\sim 3.4$ au (Fig. $4 \mathrm{i}$ ). Rosetta was at a cometocentric distance of $\sim 26.9 \mathrm{~km}$ during the 


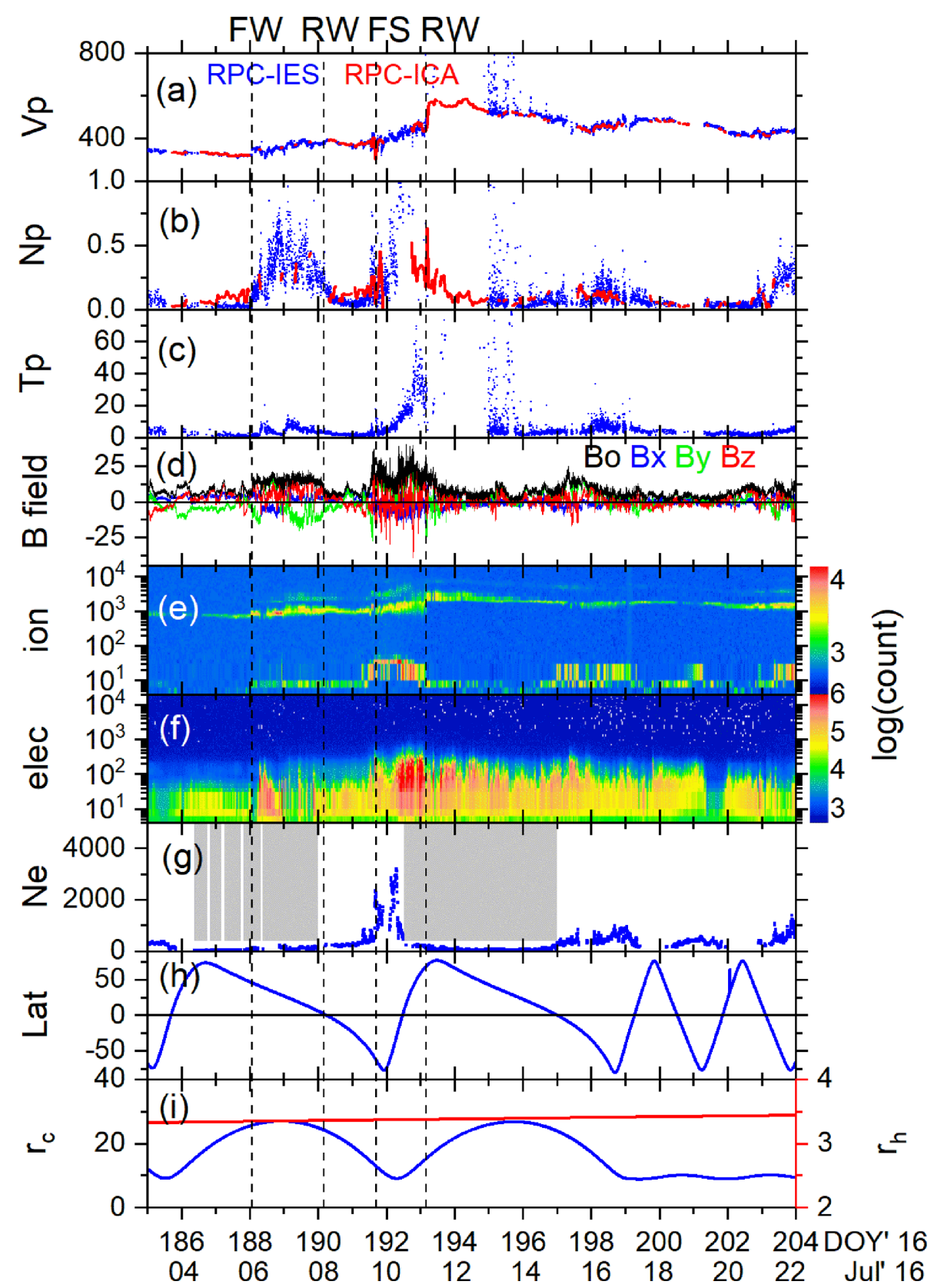

Figure 4. RPC observations for CIR during 2016 July 6-11. The panels are in the same format as in Fig. 1.

first part of the double CIR, while it approached as close as $\sim 9 \mathrm{~km}$ to $67 \mathrm{P}$ during the second part. These intervals are characterized by magnetic field compressions from $\sim 7-15$ to $\sim 22 \mathrm{nT}$, and from $\sim 9-12$ to $\sim 40$ nT, respectively (Fig. 4 d). Double CIR signature is quite clear in the $\mathrm{Np}$ and $\mathrm{Tp}$ variations. Two distinguished regions of $\mathrm{Np}$ changes from $\sim 0.05$ to $\sim 0.75 \mathrm{~cm}^{-3}$ (relative increase by a factor of $\sim 14$ ) and from $\sim 0.04$ to $\sim 0.87 \mathrm{~cm}^{-3}$ (relative increase by a factor of $\sim 21$ ) can be observed (Fig. 4b), while Tp increased from $\sim 2 \mathrm{eV}$ to $\sim 20 \mathrm{eV}$ and $\sim 60 \mathrm{eV}$ (Fig. $4 \mathrm{c}$ ).

Interestingly, RPC-IES measurement shows that there is no significant enhancement of cometary ions during the first part of the CIR, while more negative spacecraft potential increases their observed ion energy during the second part and revealed enhanced ion fluxes (Fig. 4e). It may imply that the second part of the double CIR is more effective to disturb the cometary plasma environment.
It is also because for the first part, Rosetta was above northern hemisphere and at a cometocentric distance of $\sim 26.9 \mathrm{~km}$. On the other hand, for the second part, it was at a distance of $\sim 9 \mathrm{~km}$ and over southern hemisphere where neutral densities are higher, so enhancements in ions can be more easily detected. This is consistent with larger and long-duration ionizing electron acceleration during the latter part of the CIR (Fig. 4f). About 3-4 orders of magnitude increases were recorded in ionizing electron fluxes during the two parts of the CIR.

RPC-MIP operation mode changed between LDL (grey shadings) and SDL during the CIR interval (Fig. 4g). While the plasma density may have been $>300 \mathrm{~cm}^{-3}$ during the first part, an actual measurement could not be done as RPC-MIP was operated in LDL mode at that time. On the other hand, clear signature of plasma density enhancement can be observed at the FS impact: Ne increased 
sharply from $\sim 350 \mathrm{~cm}^{-3}$ to $\sim 3000 \mathrm{~cm}^{-3}$. However, besides the CIR/FS impact, two other factors should be taken into account: first, Rosetta was at the closest cometocentric distance of $\sim 9 \mathrm{~km}$ and second, it was in the southern hemisphere of the comet where the local outgassing rate was reported to be intense (Gasc et al. 2017; Heritier et al. 2018). These two factors may contribute to the observed plasma enhancement. This will be discussed in Section 3 .

\subsection{CIR event during 2016 August 3-6}

The third CIR impact is observed from 2016 August 3 to 2016 August 6 (day 216 to 219) between a low-speed stream with speed $\sim 410 \mathrm{~km} \mathrm{~s}^{-1}$ and an HSS with peak speed $\sim 640 \mathrm{~km} \mathrm{~s}^{-1}$ (Fig. 5). The CIR continued for $\sim 3.13 \mathrm{~d}$, bounded by an FW at $\sim 0925 \mathrm{UT}$ on day 216 and an RW at $\sim 1234$ UT on day 219 (Table 1). Comet $67 \mathrm{P}$ was at a heliocentric distance of $\sim 3.5 \mathrm{au}$, while Rosetta monitored the cometary plasma environment from a distance varying between $\sim 8.9$ and $13.4 \mathrm{~km}$ (Fig. 5i).

The CIR/FW impact is characterized by magnetic field By, Bz polarity reversal (Fig. 5d), and an adjacent high plasma density region (Fig. 5b), typical of an HCS and an HPS, respectively. The CIR region is characterized by a magnetic field compression from $\sim 8-13$ to $33 \mathrm{nT}$ (Fig. 5d) and a plasma compression from Np $\sim 0.02$ to $0.6 \mathrm{~cm}^{-3}$ (Fig. $5 \mathrm{~b}$ ). A Tp change from $\sim 2.5 \mathrm{eV}$ to a peak value of $\sim 35 \mathrm{eV}$ was estimated from the RPC-IES ion distribution measurement (Fig. 5c). As in previous cases, CIR/FW is characterized by enhancements in the ionizing electron fluxes by $\sim 3$ orders of magnitude, continuing $\sim 2 \mathrm{~d}$ after the end of the CIR (Fig. $5 f$ ).

After the CIR/FW impact, RPC-MIP was operated in LDL mode (grey shading), thus any plasma density $>300 \mathrm{~cm}^{-3}$ could not be measured during those time intervals (Fig. 5g). Afterwards, a $\mathrm{Ne}$ value of $\sim 2500 \mathrm{~cm}^{-3}$ was recorded at $\sim 1507$ UT on day 217 when Rosetta was in the northern hemisphere, and it was as high as $\sim 5400 \mathrm{~cm}^{-3}$ at $\sim 0136$ UT on day 219 when Rosetta was at the southernmost latitude $\left(-79.5^{\circ}\right.$, Fig. $\left.5 \mathrm{~h}\right)$ and a cometocentric distance of $\sim 8.4 \mathrm{~km}$ (Fig. 5i). These Ne values are significantly larger than the pre-CIR values around $\sim 200-350 \mathrm{~cm}^{-3}$. However, as mentioned in Section 2.3, the Ne increases during the CIR are also influenced by the variations in the cometocentric distance and latitude of observation.

\subsection{CIR event during 2016 September 1-3}

Fig. 6 shows the RPC observations during the fourth CIR impact during 2016 September 1-3 (days 245-247) between a lowspeed stream with speed $\sim 400 \mathrm{~km} \mathrm{~s}^{-1}$ and an HSS with peak Vp $\sim 590 \mathrm{~km} \mathrm{~s}^{-1}$. Bounded by an FW at $\sim 0129$ UT on day 245 and an RW at $\sim 0623$ UT on day 247 (Table 1), the CIR had a total duration of $\sim 2.21 \mathrm{~d}$.

During this CIR interval, 67P was at a heliocentric distance of $\sim 3.7 \mathrm{au}$, and the cometocentric distance of Rosetta varied between $\sim 4$ and $16 \mathrm{~km}$ (Fig. 6i). The magnetic field amplitude Bo increased from a value of $\sim 9-14$ to $\sim 30 \mathrm{nT}$, proton density $\mathrm{Np}$ attained a peak value of $\sim 0.7 \mathrm{~cm}^{-3}$ from a pre-CIR value of $\sim 0.04 \mathrm{~cm}^{-3}$, and Tp changed from $\sim 7$ to $60 \mathrm{eV}$. The ionizing electrons exhibited flux enhancements by $\sim 3$ orders of magnitude after the CIR/FW impact.

During the entire CIR interval, Rosetta was in the southern hemisphere, and it moved closer to the comet nucleus. These two factors along with the CIR impact resulted in significant cometary plasma density increase to $\sim 2500-4000 \mathrm{~cm}^{-3}$ from the pre-CIR densities of $\sim 200-1000 \mathrm{~cm}^{-3}$ (Fig. $6 \mathrm{~g}$ ).

\section{DISCUSSION}

\subsection{Interplanetary CIR characteristics near comet 67P at large heliocentric distances}

The solar wind is very dynamic, characterized by transient impulsive events such as CMEs and CIRs that can impact on the cometary plasma dynamics. The CIRs near the comet $67 \mathrm{P}$ at heliocentric distances of $\sim 3-3.8$ au are found to have well-defined interplanetary structures with solar wind proton density and temperature enhancements, and magnetic field compressions at the low-speed stream and HSS interface region. The leading antisolar edges of the CIRs are characterized by increase in magnetic field magnitude accompanied by corresponding increases in proton speed, density, and temperature. The magnetic field decrease at the trailing edges of the CIRs is accompanied by an increase in proton speed and by decreases in proton density and temperature. Out of 10 interplanetary discontinuities at the CIR boundaries, four are determined to be forward waves and five are reverse waves, propagating at $\sim 5-92$ per cent of the magnetosonic speed at angles of $\sim 20^{\circ}-87^{\circ}$ relative to ambient magnetic field. Only one is identified to be a quasi-parallel forward shock with MMN of 1.48 and shock normal angle of $41^{\circ}$.

It may be noted that fast shocks compress both the magnetic field and plasma density by a factor of approximately the magnetosonic Mach number (for perpendicular shocks, see Kennel et al. 1985) up to a value of $\sim 4$. Since the interplanetary discontinuities detected near the comet (present study) are identified to be waves (they could have been shocks at one time) and one shock with a low Mach number, they cannot efficiently compress the near-cometary plasma and magnetic field. This suggests that the mild magnetic filed compressions (by factor of $\sim 1-4$ ) and huge plasma density increases can hardly be related to the waves at the CIR boundaries.

The leading edges of the CIRs are associated with a magnetic field polarity reversal (HCS) accompanied by a high-density cold plasma region (HPS) in the low-speed edge of the CIRs. The July CIR event (Fig. 4), which we called a 'double CIR event', was a complex event showing possible interaction between two CIRs. This structure is suggested to be associated with kinematic interaction between two HSSs with slightly different speeds and longitudinal extents (sizes) or their coalescence (see Burlaga et al. 1990, and references therein for more details on this topic).

\subsection{What is the cometary plasma response to the CIR impact?}

As shown in the previous section (Section 2) and in previous studies (Edberg et al. 2016; Galand et al. 2016), the cometary plasma density is largely dependent on the cometary latitude $(\lambda)$, longitude $(\theta)$, and on the cometocentric distance $\left(r_{\mathrm{c}}\right)$. Therefore, in order to quantify the CIR impacts on the cometary plasma density variation, the cometary ionosphere dependence on these parameters should first be isolated and removed. First, regarding cometocentric distances, the near-cometary plasma density is shown to follow a $r_{c}^{-1}$ variation (Edberg et al. 2015; Heritier et al. 2017). Second, regarding latitude and longitude variations, it is suggested that during the last four months of the Rosetta mission operation, considered for this work, the local outgassing rate was most intense over the southern hemisphere where $\mathrm{CO}_{2}$ was a major species, while $\mathrm{H}_{2} \mathrm{O}$ dominated over the northern hemisphere (Gasc et al. 2017; Heritier et al. 2018). Indeed, $\mathrm{CO}_{2}$ has a lower sublimation temperature compared to that of $\mathrm{H}_{2} \mathrm{O}$.

Following these considerations, the near-cometary plasma density $\mathrm{Ne}$ can be expressed as: $\mathrm{Ne}\left(\lambda, \theta, r_{c}\right)=\left(1 / r_{c}\right) \mathrm{Ne}^{\prime}(\lambda, \theta)$. We 


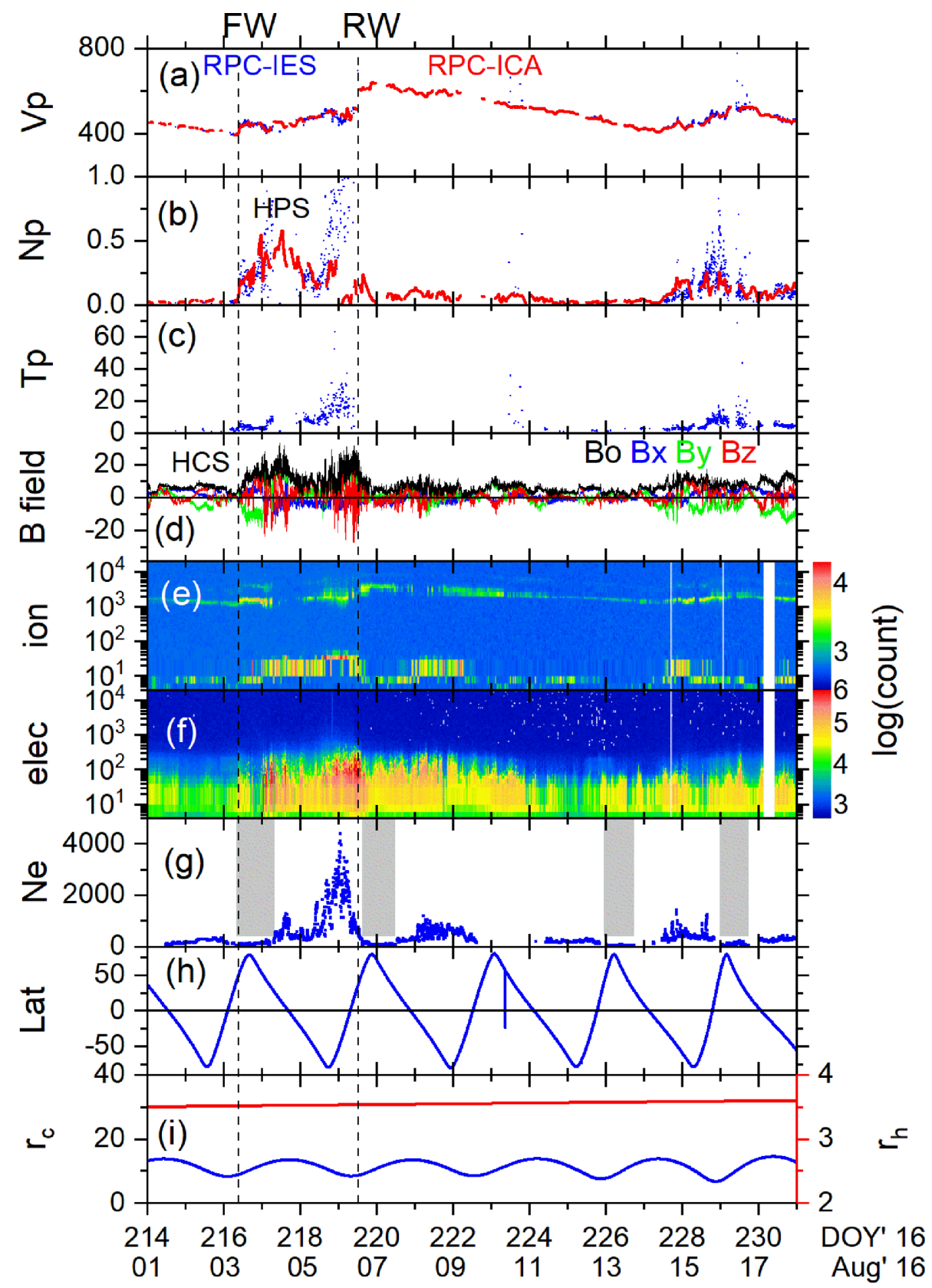

Figure 5. RPC observations for CIR during 2016 August 3-6. The panels are in the same format as in Fig. 1.

consider four months of plasma density measurements excluding the intervals with CIR impacts to generate an average $\lambda-\theta$ map of $\mathrm{Ne}$ normalized to a given cometocentric distance $r_{\mathrm{c}}$, using RPCMIP measurements. Fig. 7a shows such Ne map depicting the average Ne variation at a cometocentric distance $r_{\mathrm{c}}=10 \mathrm{~km}$. A clear hemispheric dependence can be observed with southern hemisphere exhibiting larger density $\left(\sim 1200 \mathrm{~cm}^{-3}\right)$ compared to the northern hemisphere $\left(\sim 100 \mathrm{~cm}^{-3}\right)$. This is consistent with the more intense outgassing rate observed in the southern hemisphere (Gasc et al. 2017; Heritier et al. 2018). This can be taken as the 'quiet time' cometary ionosphere density reference, that we will use to assess the CIR impact on the undisturbed cometary plasma. Such an average map may provide useful information about the degree of disturbance, by comparing the CIR time data to the average pattern.
Following the same procedure, $\lambda-\theta$ Ne maps were also created for the CIR intervals (not shown). To extract the CIR contribution on the cometary plasma variation, we estimated the Ne relative differences between the CIR periods and the quiet time period taken as a reference. Figs $7 b$, c, and d show the percentage relative difference maps during the three CIR intervals, 2016 July 6-11, 2016 August 3-6, and 2016 September 1-3, respectively. Because of the lack of Ne measurements during the first CIR interval (June 13-15), we were not able to perform this study for this CIR. The difference maps are meant to approximate the 'global' cometary plasma response to the CIRs. The peak Ne enhancements during the three CIRs are $\sim 950, \sim 2230$, and $\sim 1450$ per cent, respectively, while $\sim 500-1000$ per cent increases can be observed commonly in both hemispheres during the three events. During the July and August 


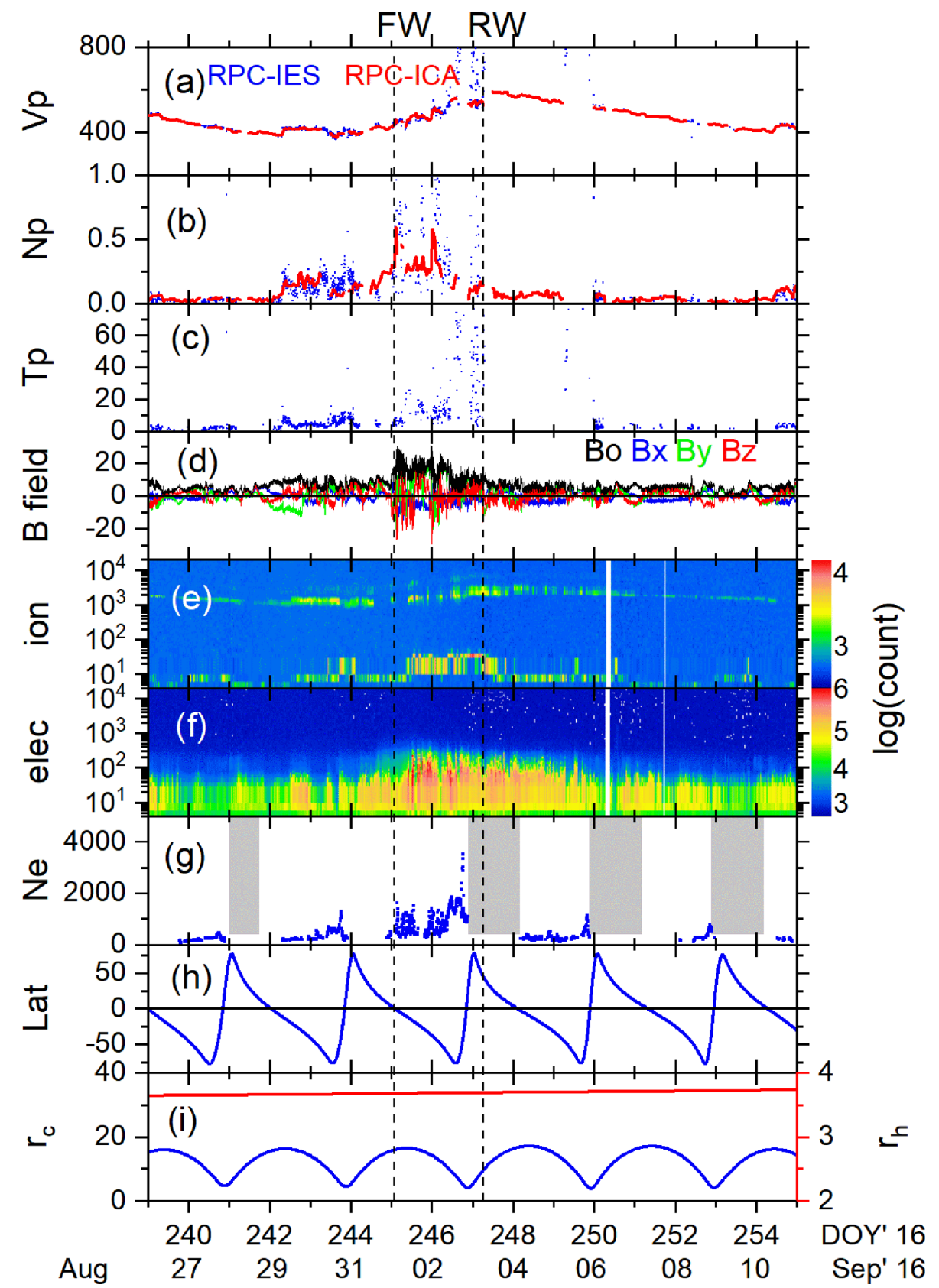

Figure 6. RPC observations for CIR during 2016 September 1-3. The panels are in the same format as in Fig. 1.

CIRs, Rosetta flew over both hemispheres. No apparent hemispheric dependence of the CIR impact on Ne was observed.

These CIR time cometary enhancements ( 500-1000 percent) are significantly large compared with CIR impacts on the Earth's ionosphere. For example, Mannucci et al. (2015) produced global differential maps of ionospheric total electron content (TEC) during two CIR impact intervals. TEC is defined as the total number of free thermal electrons present in a column of one meter squared cross section along a path between a radio transmitter and a receiver. This is an important ionospheric state parameter (see Browne et al. 1956; Evans 1956; Chakraborty \& Hajra 2008; Hajra 2012; Hajra et al. 2016a, and references therein). While large latitudinal/longitudinal variations were observed, peak TEC increases varied around 80100 percent during the CIRs. Hajra et al. (2017a) explored the altitude profile of the ionospheric $\mathrm{Ne}$ at a near-equatorial observatory Arecibo (geomagnetic: $28.17^{\circ} \mathrm{N}, 5.88^{\circ} \mathrm{E}$, dip: $46.7^{\circ}, \mathrm{L}=1.3$ ). While no data was available during a CIR event, peak Ne enhancement by a factor of $\sim 4$ or an $\sim 300$ per cent increase was recorded during an HSS interval. It should be remembered that the Earth's heliocentric distance is $\sim 1 / 3$ to $1 / 4$ of that of comet $67 \mathrm{P}$ under this study. This comparison highlights the distinguished characters of the solar wind interactions with the Earth, shielded by its own magnetosphere, and of unmagnetized solar system bodies such as comets. 


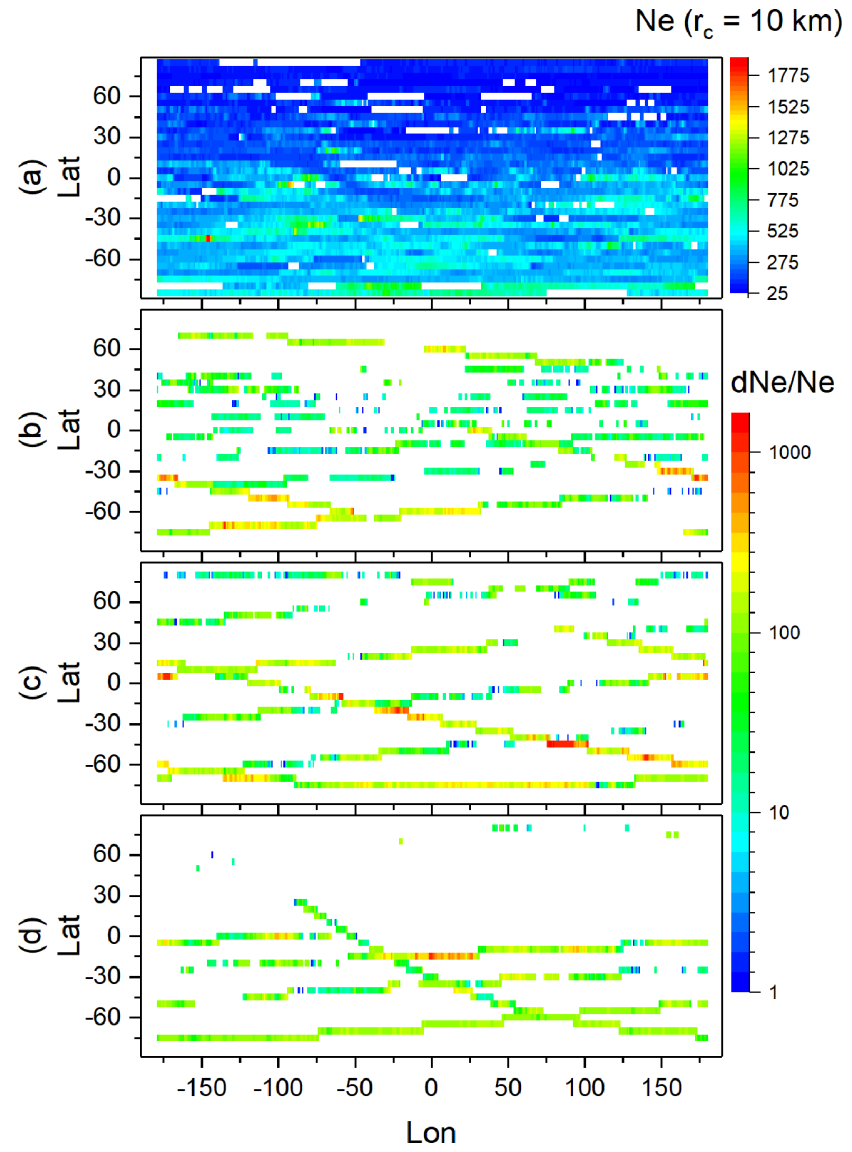

Figure 7. (a) Average quiet $\mathrm{Ne}$ map (in $\mathrm{cm}^{-3}$ ) at $r_{\mathrm{c}}=10 \mathrm{~km}$. (b)-(d) $\mathrm{Ne}$ relative difference maps (in per cent) of the CIR periods during 2016 July 6-11, 2016 August 3-6, and 2016 September 1-3, respectively, compared with the quiet times.

\subsection{What is the source of the large cometary plasma enhancement observed during the CIRs?}

Four different mechanisms were suggested by Edberg et al. (2016) to explain the cometary plasma enhancements during the CIRs in pre-perihelion phase of comet $67 \mathrm{P}$ at distances $\sim 3$ au. They are: (i) a compression of the cometary plasma by the increased solar wind ram pressure, (ii) an increase in charge exchange with solar wind protons, (iii) an acceleration of mass-loaded plasma back to the comet ionosphere, and (iv) an increase in electron-impact ionization. In this section, we will compare the relative importances of each of these mechanisms.

Regarding the contribution of the charge exchange mechanism (ii), we estimated the ion production rate of water ions produced by charge transfer of the solar wind protons and alpha particles with water neutral molecules. First, the ion particle fluxes were calculated using the density and velocity moments of the species' ion distribution functions derived from RPC-ICA. Then we obtained the ionization frequency by multiplying the solar wind ion fluxes with the cross-sections for the relevant charge transfer reactions at the average energy of the solar wind, measured in situ by RPCICA. The reactions taken into account here were single and double charge transfers, for completeness, though single charge transfer cross-sections are larger at typical solar wind energies. The reactions are $\left(\mathrm{H}^{+}, \mathrm{H}_{2} \mathrm{O}\right) \rightarrow\left(\mathrm{H}, \mathrm{H}_{2} \mathrm{O}^{+}\right),\left(\mathrm{He}^{2+}, \mathrm{H}_{2} \mathrm{O}\right) \rightarrow\left(\mathrm{He}^{+}, \mathrm{H}_{2} \mathrm{O}^{+}\right),(\mathrm{He}$, $\mathrm{H}_{2} \mathrm{O}^{2+}$ ), and $\left(\mathrm{He}^{+}, \mathrm{H}_{2} \mathrm{O}\right) \rightarrow\left(\mathrm{He}, \mathrm{H}_{2} \mathrm{O}^{+}\right)$(see Simon Wedlund et al.

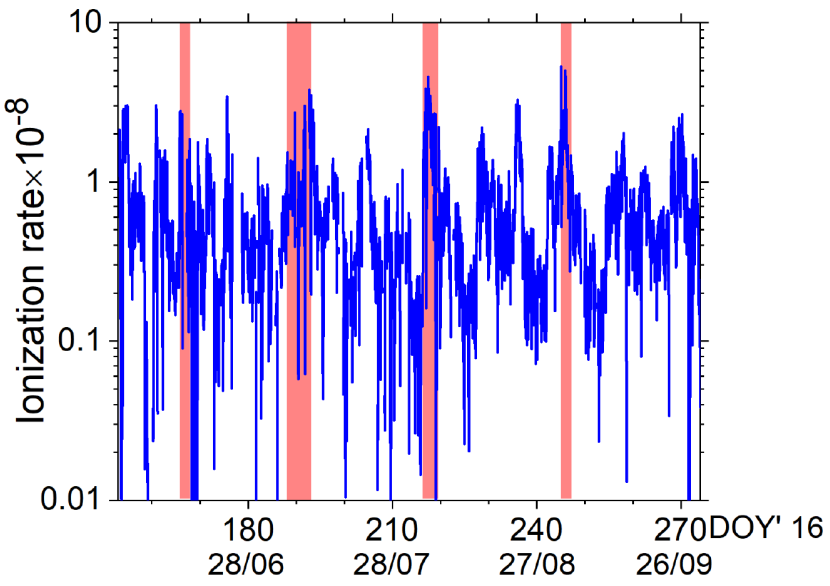

Figure 8. Average total ion production rate (in $\mathrm{s}^{-1}$ ) due to solar wind charge transfer during 2016 June through September. The light red shaded regions show the CIR impact intervals as in Fig. 1.

2016, 2017, for more details on the cross sections). The average $(1 \mathrm{~h})$ total ion production rate due to solar-wind charge transfer is shown in Fig. 8. It peaks at about $4-5 \times 10^{-8} \mathrm{~s}^{-1}$ during the CIR impacts on the cometary environment. Such levels of ionization are not uncommon throughout the 2016 June-September period, and are likely associated with a combination of neutral atmosphere and solar wind variations.

Regarding the contribution of photoionization and electronimpact ionization (iv), we use a multi-instrumental data-based ionospheric model by Galand et al. (2016), which suggests that the cometary plasma density variation is mainly controlled by these two mechanisms, at least in the inner cometary ionosphere unperturbed from solar wind transient events. The model uses time-varying electron-impact ionization frequency based on RPCIES measurements, photoionization frequency obtained from Thermosphere Ionosphere Mesophere Energetics and Dynamics-Solar EUV Experiment (TIMED-SEE, Woods et al. 2005) and interpolated to comet $67 \mathrm{P}$ along with cometary neutral density and neutral composition measured by the ROSINA-COPS (Rosetta Orbiter Spectrometer for Ion and Neutral Analysis-COmet Pressure Sensor) and ROSINA-DFMS (Double Focusing Mass Spectrometer) instruments (Balsiger et al. 2007), respectively. The respective contributions of photoionization and electron-impact ionization are reported to be highly variable (see Galand et al. 2016; Heritier et al. 2017; Hajra et al. 2017b).

The Galand et al. (2016) model study was extended by Heritier et al. (2018) in order to identify the ionospheric drivers throughout the two years of the 67P escort phase by Rosetta and to assess their variability over time and heliocentric distance (before and after perihelion). Fig. 9 shows an example during the August CIR event, based on Heritier et al. (2018). The average ionizing electron density increases from $\sim 200$ to $3000 \mathrm{~cm}^{-3}$ during the CIR (Fig. 9a). This leads to a 2 orders of magnitude increase in electron-impact ionization to $\sim 3.5 \times 10^{-6} \mathrm{~s}^{-1}$, while the photoionization frequency is significantly low $\left(\sim 4 \times 10^{-8} \mathrm{~s}^{-1}\right)$ (Fig. 9b). We note also that the electron-impact ionization frequency (Fig. 9b) is about 2 orders of magnitude higher compared to the charge exchange ion production frequency (Fig. 8) at the position of Rosetta. Fig. 9c shows a comparison between the cometary plasma density derived from the RPC-MIP measurement and the modelled ionospheric densities. On the one hand, ionospheric density due to photoionization only is insignificant compared to the actual plasma density. On the other 


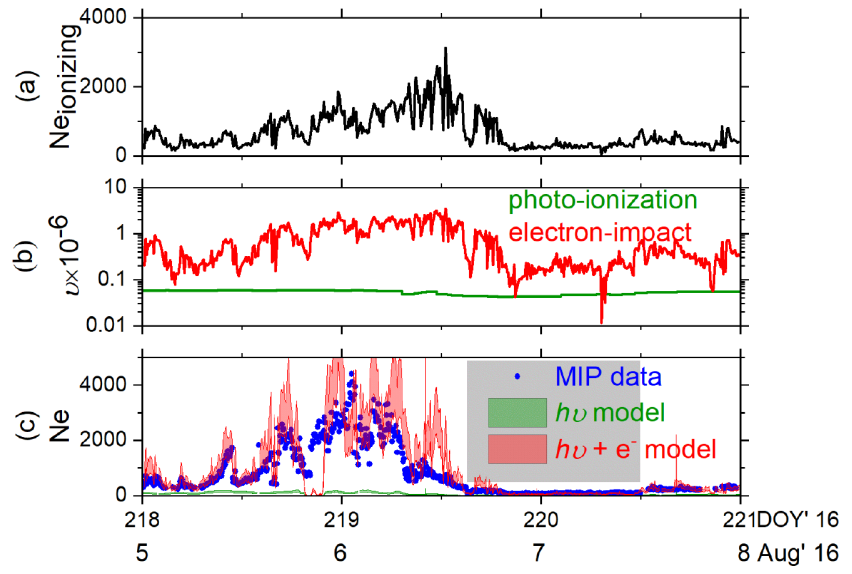

Figure 9. (a) Average ionizing electron density (in $\mathrm{cm}^{-3}$ ), (b) photoionization frequency (in $\mathrm{s}^{-1}$, green curve) and electron-impact ionization frequency corrected with spacecraft potential (in $\mathrm{s}^{-1}$, red curve), (c) electron number density estimated from RPC-MIP (in $\mathrm{cm}^{-3}$, blue dots), modelled ionospheric densities (in $\mathrm{cm}^{-3}$ ) using photoionization only (green) and both photoionization and electron-impact ionization (red), assuming outflow velocity from $400 \mathrm{~m} \mathrm{~s}^{-1}$ (upper bound) to $700 \mathrm{~m} \mathrm{~s}^{-1}$ (lower bound) during 2016 August $5-8$. The grey shading indicates inaccessible densities associated with measurements limits in LDL operational mode as in Fig. 1.

hand, the total density due to photoionization and electron-impact ionization matches well the cometary plasma density variations observed during the CIRs. This implies that while photoionization (and charge exchange) had a very minor contribution to the cometary plasma, electron-impact ionization was found to be the dominating ion source during the CIRs. Large ionizing electron flux enhancements during the CIRs (as observed in this study and previously in Edberg et al. (2016) led to a significant increase in the electron-impact ionization rate. ${ }^{1}$

The relative contributions of the mechanism (iii), i.e. an acceleration of mass-loaded plasma to the comet ionosphere, cannot be estimated directly. On the other hand, as argued previously, the mild magnetic field compressions and huge plasma density increases are unlikely related to the observed waves at the CIR boundaries (mechanism i). However, this study, combined with Heritier et al. (2018), identifies the increase in electron-impact ionization as the dominating mechanism explaining the large increase in cometary plasma during the CIRs, at least in the cases studied here. The other three mechanisms proposed by Edberg et al. (2016) may only play minor roles in the cometary plasma density variation during the CIR events.

A final point that remains to be understood is the source mechanism of the ionizing electrons during the CIR events. At present time, their origin is not fully understood. During periods without CIRs, Broiles et al. (2016) suggested that energetic electrons observed at comet $67 \mathrm{P}$ could be generated through wave-particle

\footnotetext{
${ }^{1}$ It may be noted, however, that the ionization model fails to reproduce the observed plasma density at $\sim 1230$ UT on August 6 (Fig. 9c). This is associated with the ionizing electron density retrieved from RPC-IES (Fig. 9a) at that time, and that appears larger than the total Ne observed by RPC-MIP. This peculiarity is observed during $\sim 1 \mathrm{~h}$, and is not currently understood. This is not the case during the rest of the analysis. It may be due to some atypical electron distributions. The estimations of the ionizing density assume an isotopic distribution. This can yield an overestimation when the electron population has more a beam-like distribution, which may occur during CIRs.
}

interactions involving lower hybrid waves, while Madanian et al. (2016) suggested that they could be generated through the effect of the cometary ambipolar electric field. Moreover, Particle-In-Cell simulations including the electron kinetic physics showed that the potential well associated with the cometary ambipolar electric field should accelerate electrons of solar wind origin up to ionizing energies in the near-cometary environment (Deca et al. 2017). Further simulation studies are required to identify the source of energetic electrons during CIR impacts. This is left for a future work.

\section{SUMMARY}

We have presented a quantitative study on the interplanetary characteristics, evolution, and impacts of the CIRs in a near-cometary plasma environment. The comet 67P plasma and magnetic field variations at large heliocentric distances of $\sim 3-3.8$ au measured in situ by the Rosetta spacecraft at cometocentric distances of $\sim 4-30 \mathrm{~km}$ are explored to study the cometary plasma response to four CIR events during 2016 June through September. The CIRs were identified in the interface region between low-speed solar winds and HSSs with peak proton speeds of $\sim 320-400 \mathrm{~km} \mathrm{~s}^{-1}$ and $\sim 580$ $640 \mathrm{~km} \mathrm{~s}^{-1}$, respectively, measured near comet $67 \mathrm{P}$. The main results of this work may be summarized as follows:

(i) The near-cometary, interplanetary CIRs are characterized by relative increases (i.e. increases from the pre-CIR values divided by the pre-CIR values) in magnetic field intensity by factors of $\sim 1$ 4 , in solar wind proton density by $\sim 13-29$ and in temperature by $\sim 7-29$.

(ii) The CIRs are bounded by interplanetary discontinuities. At the CIR leading edges an increase in magnetic field intensity is accompanied by increases in solar wind proton speed, density, and temperature. The trailing edges of the CIRs are characterized by magnetic field decreases accompanied by an increase in proton speed and by decreases in proton density and temperature.

(iii) Out of 10 interplanetary discontinuities at the CIR boundaries detected in this study, four are determined to be forward waves and five are reverse waves, propagating at $\sim 5-92$ percent of the magnetosonic speed at angles of $\sim 20^{\circ}-87^{\circ}$ relative to ambient magnetic field. Only one is identified to be a quasi-parallel forward shock with magnetosonic Mach number of 1.48 and shock normal angle of $41^{\circ}$.

(iv) The 'double CIR event' of 2016 July shows evidence of possible kinematic interactions between two HSSs/CIRs at large heliocentric distances.

(v) During the CIR impacts, ionizing (>12-200 eV) electrons exhibit increases in fluxes by $\sim 3-4$ orders of magnitude. The flux enhancements are long-duration, continuing for $\sim 1.5-2 \mathrm{~d}$ after the CIRs terminate. Large and long-duration cometary ionizing electron flux enhancements seem to be a good proxy indication of the nearcometary CIR impact.

(vi) The CIR impact on the cometary plasma density was quantified by identifying and removing the quiet time plasma dependence on the cometary latitude, longitude, and the cometocentric distance. Cometary plasma density exhibited large increases of 500-1000 per cent of the quiet time reference values.

(vii) During the CIRs, the ion production rate due to solar wind charge exchange was observed to be $\sim 2$ orders of magnitude lower compared to the electron-impact ionization frequency.

(viii) Multi-instrument analysis of the cometary ionosphere (Heritier et al. 2018) reveals that photoionization process contributes insignificantly to the cometary plasma variation during the CIRs, 
while electron-impact ionization is the dominating process. Increased electron-impact ionization frequency, resulting from an enhancement in the energetic electron fluxes, is suggested to be the prime cause of the cometary plasma enhancements during the CIRs.

\section{FINAL COMMENTS}

In this work, we explored the near-cometary plasma response to CIRs during the post-perihelion phase of its solar orbit, while the previous study by Edberg et al. (2016) corresponded to the pre-perihelion phase. In both cases, the comet was at heliocentric distances of $\sim 3$ au, characterized by a weak-to-moderate outgassing rate. The CIR impacts on the cometary plasma are found to be qualitatively identical in both pre- and post-perihelion phases. Large plasma enhancements, long-duration ionizing electron flux increases, and magnetic field intensifications can be considered as the 'typical CIR impact signatures' in the near-cometary environment at large heliocentric distances. However, the plasma density increases vary from one case to the other depending on the distance from the comet nucleus, latitude, and longitude. During the observations reported in this study, Rosetta was located in the weakly outgassing cometary plasma, so that the passage of a CIR can unambiguously be identified from the solar wind ion measurements near the comet. On the other hand, during highly active comet phases, i.e. near perihelion, a so-called solar wind ion cavity builds up around the comet nucleus (Nilsson et al. 2017) where solar wind ions cannot penetrate anymore. In such a case the unambiguous detection of CIR impacts from Rosetta measurements is expected to be much more difficult to achieve. The investigation of CIR impacts on the plasma environment of an active comet will be considered in future works, based on the knowledge acquired from CIR impacts on the plasma environment of a low-activity comet (present study).

\section{ACKNOWLEDGEMENTS}

Rosetta is a European Space Agency (ESA) mission with contributions from its member states and National Aeronautics and Space Administration (NASA). The work at LPC2E/CNRS was supported by CNES, ESEP, and by ANR under the financial agreement ANR15-CE31-0009-01. Work at Imperial College London is supported by Imperial College President's scholarship, STFC of UK under grants ST/K001051/1 and ST/N000692/1 and ESA under contract No.4000119035/16/ES/JD. The work at University of Oslo is supported by the Research Council of Norway grant No. 240000. The work on RPC-MAG was financially supported by the German Ministerium für Wirtschaft und Energie and the Deutsches Zentrum für Luft- und Raumfahrt under contract 50QP 1401. Portions of the research were conducted at the Jet Propulsion Laboratory, California Institute of Technology, under contract with NASA. We acknowledge ISSI for the opportunity it offered for valuable discussions on this topic as part of the International Team 'Plasma Environment of comet 67P after Rosetta'. This work has used AMDA and RPC quick-look data base to provide an initial overview of the event studied. This is provided through a collaboration between the Centre de Données de la Physique des Plasmas (supported by CNRS, CNES, Observatoire de Paris, and Université Paul-Sabatier, Toulouse) and Imperial College London (supported by the UK Science and Technology Facilities Council). The data used in this paper will soon be made available on the ESA Planetary Science Archive (PSA) and is available upon request until that time.

\section{REFERENCES}

Abraham-Shrauner B., 1972, J. Geophys. Res., 77, 736

Balogh A. et al., 1999, Space Sci. Rev., 89, 141

Balsiger H. et al., 2007, Space Sci. Rev., 128, 745

Bartels J., 1932, Terr. Magn. Atmos. Electr., 37, 1

Bartels J., 1934, Terr. Magn. Atmos. Electr., 39, 201

Behar E., Nilsson H., Alho M., Goetz C., Tsurutani B., 2017, MNRAS, 469, S396

Broiles T. W. et al., 2016, MNRAS, 462, S312

Browne I. C., Evans J. V., Hargreaves J. K., Murray W. A. S., 1956, Proc. Phys. Soc. Sec. B, 69, 901

Burch J. L., Goldstein R., Cravens T. E., Gibson W. C., Lundin R. N., Pollock C. J., Winningham J. D., Young D. T., 2007, Space Sci. Rev., 128, 697

Burlaga L. F., Mish W. H., Whang Y. C., 1990, J. Geophys. Res., 95, 4247

Carr C. et al., 2007, Space Sci. Rev., 128, 629

Chakraborty S. K., Hajra R., 2008, Ann. Geophys., 26, 47

Chao J. K., 1973, J. Geophys. Res., 78, 5411

Churyumov K. I., Gerasimenko S. I., 1972, in Chebotarev G. A., Kazimirchak-Polonskaya E. I., Marsden B. G., eds, IAUS, Vol. 45, The Motion, Evolution of Orbits, and Origin of Comets. Springer Netherlands, Dordrecht, p. 27

Collard H. R., Wolfe J. H., 1974, in, Solar wind three. University of California, Los Angeles, p. 281

Deca J., Divin A., Henri P., Eriksson A., Markidis S., Olshevsky V., Horányi M., 2017, Phys. Rev. Lett., 118, 205101

Dubinin E. et al., 2008, Geophys. Res. Lett., 35, L11103

Dungey J. W., 1961, Phys. Rev. Lett., 6, 47

Echer E., Tsurutani B. T., Guarnieri F. L., 2010, Adv. Space Res., 45, 798

Edberg N. J. T. et al., 2011, J. Geophys. Res., 116

Edberg N. J. T. et al., 2015, Geophys. Res. Lett., 42, 4263

Edberg N. J. T. et al., 2016, J. Geophys. Res., 121, 949

Eriksson A. I. et al., 2007, Space Sci. Rev., 128, 729

Evans J. V., 1956, Proc. Phys. Soc. Sec. B, 69, 953

Galand M. et al., 2016, MNRAS, 462, S331

Gasc S. et al., 2017, MNRAS, 469, S108

Glassmeier K.-H., Boehnhardt H., Koschny D., Kührt E., Richter I., 2007a, Space Sci. Rev., 128, 1

Glassmeier K.-H. et al., 2007b, Space Sci. Rev., 128, 649

Guarnieri F. L., Tsurutani B. T., Vieira L. E. A., Hajra R., Echer E., Mannucci A. J., Gonzalez W. D., 2018, Nonlinear Processes in Geophysics, 25, 67

Hajra R., 2012, PhD thesis, University of Calcutta

Hajra R., Tsurutani B. T., 2018a, in Buzulukova N., ed., Extreme Events in Geospace: Origins, Predictability, and Consequences, Elsevier, Netherlands, p. 373

Hajra R., Tsurutani B. T., 2018b, ApJ, 858, 123

Hajra R., Echer E., Tsurutani B. T., Gonzalez W. D., 2013, J. Geophys. Res., 118,5626

Hajra R., Tsurutani B. T., Echer E., Gonzalez W. D., 2014a, Geophys. Res. Lett., 41, 1876

Hajra R., Echer E., Tsurutani B. T., Gonzalez W. D., 2014b, J. Geophys. Res., 119, 2675

Hajra R., Echer E., Tsurutani B. T., Gonzalez W. D., 2014c, J. Atmos. Sol. Terr. Phys., 121, 24

Hajra R., Tsurutani B. T., Echer E., Gonzalez W. D., Brum C. G. M., Vieira L. E. A., Santolik O., 2015a, Earth Planets Space, 67, 109

Hajra R., Tsurutani B. T., Echer E., Gonzalez W. D., Santolik O., 2015b, ApJ, 799, 39

Hajra R., Chakraborty S. K., Tsurutani B. T., DasGupta A., Echer E., Brum C. G. M., Gonzalez W. D., Sobral J. H. A., 2016a, J. Space Weather Space Clim., 6, A29

Hajra R., Tsurutani B. T., Echer E., Gonzalez W. D., Gjerloev J. W., 2016b, J. Geophys. Res., 121, 7805

Hajra R., Tsurutani B. T., Brum C. G. M., Echer E., 2017a, Geophys. Res. Lett., 44, 7607

Hajra R. et al., 2017b, A\&A, 607, A34

Heritier K. L. et al., 2017, MNRAS, 469, S118 
Heritier K. L. et al., 2018, Plasma source and loss at comet 67P during the Rosetta mission, A\&A. doi: 10.1051/0004-6361/201832881

Intriligator D. S., Neugebauer M., 1975, J. Geophys. Res., 80, 1332

Kennel C. F., Edmiston J. P., Hada T., 1985, A Quarter Century of Collisionless Shock Research. American Geophysical Union, Washington, D.C., p. 1

Madanian H. et al., 2016, J. Geophys. Res., 121, 5815

Mannucci A. J. et al., 2015, Space Weather, 13, 125

Mendes O., Domingues M. O., Echer E., Hajra R., Menconi V. E., 2017, Nonlinear Processes in Geophysics, 24, 407

Ness N. F., Wilcox J. M., 1964, Phys. Rev. Lett., 13, 461

Neugebauer M., Snyder C. W., 1966, J. Geophys. Res., 71, 4469

Newton H. W., Nunn M. L., 1951, MNRAS, 111, 413

Nilsson H. et al., 2007, Space Sci. Rev., 128, 671

Nilsson H. et al., 2017, MNRAS, 469, S252

Parker E. N., 1963, Interscience Publishers. New York

Phillips J. L. et al., 1994, Geophys. Res. Lett., 21, 1105

Pizzo V. J., 1985, in Tsurutani B. T., Stone R. G., eds, Collisionless Shocks in the Heliosphere: Reviews of Current Research, Vol. 35, American Geophysical Union, Washington, D. C., p. 51

Richter A. K., Hsieh K. C., Luttrell A. H., Marsch E., Schwenn R., 1985 Review of Interplanetary Shock Phenomena Near and within 1 AU. American Geophysical Union, Washington, D.C., 33

Simon Wedlund C. et al., 2016, MNRAS, 587, A154

Simon Wedlund C. et al., 2017, Astron. Astrophys., 604, A73

Siscoe G. L., 1972, J. Geophys. Res., 77, 27
Smith E. J., 1985, in Tsurutani B. T., Stone R., eds, Interplanetary Shock Phenomena Beyond 1 AU, Collisionless Shocks in the Heliosphere: Reviews of Current Research, AGU, Washington, D.C.

Smith E. J., Wolfe J. H., 1976, Geophys. Res. Lett., 3, 137

Smith E. J., Tsurutani B. T., Rosenberg R. L., 1978, J. Geophys. Res., 83, 717

Sonett C. P., Colburn D., 1965, Planet. Space Sci., 13, 675

Souza A. M., Echer E., Bolzan M. J. A., Hajra R., 2016, Journal of Atmospheric and Solar-Terrestrial Physics, 149, 81

Souza A. M., Echer E., Bolzan M. J. A., Hajra R., 2018, Annales Geophysicae, 36, 205

Trotignon J. G. et al., 2007, Space Sci. Rev., 128, 713

Tsurutani B. T., Gonzalez W. D., 1987, Planet. Space Sci., 35, 405

Tsurutani B. T., Lin R. P., 1985, J. Geophys. Res., 90, 1

Tsurutani B. T., Smith E. J., Pyle K. R., Simpson J. A., 1982, J. Geophys Res., 87, 7389

Tsurutani B. T., Ho C. M., Arballo J. K., Goldstein B. E., Balogh A., 1995, Geophys. Res. Lett., 22, 3397

Tsurutani B. T. et al., 2006, J. Geophys. Res., 111, A07S01

Tsurutani B. T., Lakhina G. S., Verkhoglyadova O. P., Gonzalez W. D., Echer E., Guarnieri F. L., 2011, J. Atmos. Sol. Terr. Phys., 73, 5

Winterhalter D., Smith E. J., Burton M. E., Murphy N., McComas D. J., 1994, J. Geophys. Res., 99, 6667

Woods T. N. et al., 2005, J. Geophys. Res., 110, A01312

This paper has been typeset from a $\mathrm{T}_{\mathrm{E}} \mathrm{X} / \mathrm{LT}_{\mathrm{E}} \mathrm{X}$ file prepared by the author. 